\title{
BMJ Open Long-term survival outcomes and adverse effects of nasopharyngeal carcinoma patients treated with IMRT in a non-endemic region: a population- based retrospective study
}

Man Xu, ${ }^{1,2}$ Jian Zang, ${ }^{3}$ Shanquan Luo, ${ }^{3}$ Jianhua Wang, ${ }^{3}$ Xuqi Li (1) ${ }^{1}$

To cite: Xu M, Zang J, Luo S, et al. Long-term survival outcomes and adverse effects of nasopharyngeal carcinoma patients treated with IMRT in a non-endemic region: a population-based retrospective study. BMJ Open 2021;11:e045417. doi:10.1136/ bmjopen-2020-045417

- Prepublication history for this paper is available online. To view these files, please visit the journal online (http://dx.doi. org/10.1136/bmjopen-2020045417).

MX and JZ contributed equally.

Received 13 October 2020 Accepted 09 July 2021

Check for updates

(C) Author(s) (or their employer(s)) 2021. Re-use permitted under CC BY-NC. No commercial re-use. See rights and permissions. Published by BMJ.

${ }^{1}$ Department of Gengral Surgery, The First Affiliated Hospital of Xi'an Jiaotong University, Xi'an, Shaanxi, China

${ }^{2}$ Department of Radiation Oncology, Xi'an Gaoxin Hospital, Xi'an, Shaanxi, China

${ }^{3}$ Department of Radiation Oncology, Air Force Medical University Xijing Hospital, Xian, Shaanxi, China

Correspondence to Dr Xuqi Li; lixuqi@163.com

\section{ABSTRACT}

Objectives To evaluate the long-term survival outcomes and adverse effects of intensity-modulated radiotherapy (IMRT) for nasopharyngeal carcinoma (NPC) and to summarise the experiences of IMRT in NPC in the past few decades in non-endemic northwest China.

Design A population-based retrospective study.

Setting An experience of using IMRT in non-endemic region of China.

Participants The study included 792 newly diagnosed and non-metastatic NPC patients who received IMRT from January 2006 to September 2018 in Xijing Hospital. Outcome measures The survival outcomes, adverse effects and failure patterns were evaluated by univariate, multivariate and subgroup analyses.

Results With a median follow-up time of 46.2 months, the 5-year local recurrence-free survival, regional recurrencefree survival, distant metastasis-free survival, diseasefree survival (DFS) and overall survival (OS) rates were $90.8 \%, 97.0 \%, 82.8 \%, 69.6 \%$ and $78.0 \%$, respectively. Multivariate analysis showed that age, $\mathrm{N}$ stage, clinical stage, pathological type and primary tumour volume of more than $23 \mathrm{~cm}^{3}$ were the independent prognosis factors for DFS (all $p<0.05$ ); age, $N$ stage, pathological type, cervical lymph node necrosis, and anaemia were significantly associated with $\mathrm{OS}$ (all $p<0.05$ ). The most common acute toxicities of IMRT were dermatitis, mucositis and dysphagia. Xerostomia and hearing impairment were the top two late toxicities. The main failure patterns were distant metastasis and local and/or regional relapses.

Conclusions Similar survival, toxicities and failure patterns have been observed in patients treated with IMRT in a non-endemic area of China when compared with that in endemic areas. Induction chemotherapy combined with concurrent chemoradiotherapy may benefit locally advanced NPC in non-endemic areas of China.

\section{INTRODUCTION}

Nasopharyngeal carcinoma (NPC) is an epithelium malignancy with a characteristic of unbalanced regional distribution. Statistics revealed that more than $70 \%$ of newly diagnosed NPCs are in east and southeast Asia. It
Strengths and limitations of this study

- Our study summarises the experiences of intensitymodulated radiotherapy in nasopharyngeal carcinoma in the past few decades in northwest non-endemic area of China.

- The clinical characteristics, survival outcomes, long-term adverse effects and failure patterns were reported.

- A large cohort study ( $\mathrm{n}=792)$ and long-term followup (46.2 months).

- This study is expected to lay the foundation for conducting future prospective study.

- The limitations of this study are that the patients are derived from a single centre and the study's retrospective design.

is prevalent in southern China, with the world age-standardised rate of approximately 3.0 per 100000 compared with 0.4 per 100000 in Western countries. ${ }^{12}$ In China, the morbidity and mortality of NPC were evidently higher in the southern area than that in the other areas while the northern area ranks the lowest. ${ }^{3}$

Radiotherapy (RT) is the primary treatment modality for NPC due to the high sensitivity of nasopharyngeal tumours to radiation. With the progression of radiation techniques, RT has changed from conventional two-dimensional conformal radiotherapy (2D-CRT) to 3D-CRT and to more advanced intensity-modulated RT (IMRT). Nowadays, IMRT is the most widely used technique in RT. Local or regional controls and survival have been improved by the parallel advantages of dosimetric properties and reduced toxicity. ${ }^{4-6}$ The 5-year locoregional relapse rate of non-metastatic NPC has been reduced to $7.4 \% .^{7}$ Furthermore, IMRT was closely related to a better 5 -year overall survival (OS) when compared with 2D-CRT or 3D-CRT, 
along with significantly reduced toxicities such as xerostomia, trismus and temporal lobe neuropathy. ${ }^{5}$ However, these data are mainly acquired from experiences in epidemic regions. To date, the literature related to the long-term survival outcomes and radiation-induced toxicities of a large cohort of patients who underwent IMRT in non-endemic regions are limited. Thus, in the current study, we intend to comprehensively evaluate the survival outcomes and adverse effects of patients treated with IMRT in a non-endemic region of China.

\section{MATERIALS AND METHODS \\ Patients}

From January 2006 to September 2018, a total of 792 patients were included in the study. The inclusion criteria were as follows: (1) patients from northwest region of China, (2) pathologically confirmed NPC, (3) previously untreated, (4) no evidence of distant metastasis, (5) no previous malignancies or other concomitant malignant diseases, and (6) received a whole course of IMRT and no molecular targeted therapy.

\section{Radiotherapy}

IMRT was delivered within 2 weeks of completion of the induction chemotherapy (IC). External megavoltage photons were used to treat primary lesions and cervical lymph nodes. The gross tumour volume (GTV) included the entire nasopharygeal tumours (GTVnx) and the positive lymph nodes of the neck (GTVnd). The clinical target volume (CTV) contained the adjacent areas at risk for microscopic disease. The high-risk CTV1 was the GTV plus the entire nasopharyngeal mucosa, retropharyngeal lymph nodes, skull base, parapharyngeal space, pterygopalatine fossa, sphenoid sinus, posterior third of the nasal cavity, and maxillary sinus. The low-risk CTV2 covered the lower neck without lymph node metastasis and supraclavicular fossa. The planning target volumes (PTVs) were delineated by adding $3 \mathrm{~mm}$ margins to the GTVs and CTVs. The prescribed radiation doses to the PTV of primary tumours (GTVnx-P) were $69.96 \mathrm{~Gy} / 33$ fractions for T1-2 disease and 72.6-74.25 Gy/33 fractions for T3-4 lesion, 66-73.92 Gy/30-33 fractions for the PTV of positive lymph nodes (GTVnd-P), 60-64 Gy/30-33 fractions for the PTV of CTV-1, 50-54 Gy/28-33 fractions for the PTV of CTV-2 (figure 1). All patients were treated with $2 \mathrm{~Gy} /$ fraction daily for five consecutive days per week. The doses for the normal tissues and organs at risk were confined below tolerance levels.

\section{Chemotherapy}

Overall, chemotherapy was administered to $93.9 \%$ of patients. The details of the chemotherapy strategy are illustrated in table 1 . The regimens for induction and adjuvant chemotherapy (AC) were docetaxel, cisplatin and fluorouracil (TPF), docetaxel and cisplatin (TP), Cisplatin and fluorouracil (PF), and gemcitabine and cisplatin (GP). The TPF regimen consisted of docetaxel

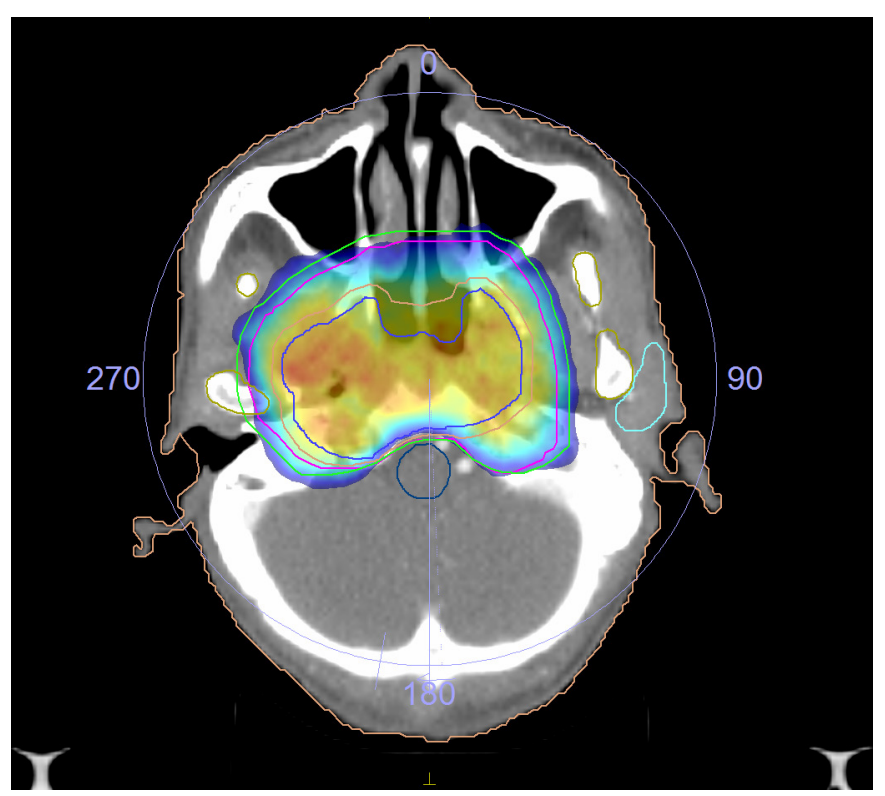

Figure 1 Target paint example.

$\left(75 \mathrm{mg} / \mathrm{m}^{2}\right)$ intravenously on day 1 , cisplatin $(75 \mathrm{mg} /$ $\mathrm{m}^{2}$ ) continuously intravenously on days $1-3$, and fluorouracil $\left(500 \mathrm{mg} / \mathrm{m}^{2}\right)$ continuously intravenously on days $1-5$. The TP regimen was administered as docetaxel (75 $\mathrm{mg} / \mathrm{m}^{2}$; intravenously) on day 1 and cisplatin (75 mg/ $\mathrm{m}^{2}$ ) continuously intravenously on day 1 . The PF regimen comprised of cisplatin $\left(75 \mathrm{mg} / \mathrm{m}^{2}\right.$; intravenously) on day 1 and fluorouracil $\left(500 \mathrm{mg} / \mathrm{m}^{2}\right)$ continuously (intravenously) on days $1-5$. The GP regimen included cisplatin (75 mg/m²; intravenously) on day 1 and gemcitabine $\left(1000 \mathrm{mg} / \mathrm{m}^{2}\right.$; intravenously) on days 1 and 8 . All the

\begin{tabular}{|c|c|}
\hline Patient characteristics & No/mean (range) \\
\hline Age (year) & $47.3(9-83)$ \\
\hline Tumour volume (mL) & $22.5(2.4-232.0)$ \\
\hline Lymph nodes size (cm) & $1.7(0.8-8.9)$ \\
\hline Gender (male/female) & $566 / 226$ \\
\hline Age ( $<50$ years $/ \geq 50$ years) & $446 / 346$ \\
\hline LDH ( $\leq 174 \mathrm{u} / \mathrm{L} />174 \mathrm{u} / \mathrm{L})$ & $567 / 225$ \\
\hline T stage (T1/T2/T3/T4) & $87 / 277 / 133 / 295$ \\
\hline N stage (N0/N1/N2/N3a/N3b) & $105 / 186 / 347 / 64 / 90$ \\
\hline Clinical stage (I/II/III/IVa/IVb) & $22 / 124 / 246 / 246 / 154$ \\
\hline WHO histology (I/II/III) & $3 / 210 / 579$ \\
\hline \multicolumn{2}{|l|}{ Diagnostic imaging technique } \\
\hline MRI & 792 \\
\hline \multicolumn{2}{|l|}{ Chemotherapy } \\
\hline $\begin{array}{l}\mathrm{RT} / \mathrm{CCRT} / \mathrm{IC}+\mathrm{CCRT} / \mathrm{IC}+\mathrm{RT} / \mathrm{CCRT}+ \\
\mathrm{AC} / \mathrm{RT}+\mathrm{AC} / \mathrm{IC}+\mathrm{CCRT}+\mathrm{AC} / \mathrm{IC}+\mathrm{RT}+\mathrm{AC}\end{array}$ & $48 / 243 / 365 / 51 / 21 / 8 / 55 / 1$ \\
\hline CNN (yes/no) & $401 / 391$ \\
\hline
\end{tabular}

AC, adjuvant chemotherapy; CCRT, concurrent chemo-radiotherapy; CNN, cervical nodal necrosis; IC, induction chemotherapy; LDH, lactic dehydrogenase; MRI, magnetic resonance imaging; RT, radiotherapy. 
regimens were repeated every 3 weeks for 2-3 cycles for IC and every 4 weeks for 2-3 cycles for AC. Concurrent chemoradiotherapy (CCRT) consisted of cisplatin-based chemotherapy that was administered as cisplatin $(40 \mathrm{mg}$ / $\mathrm{m}^{2}$; intravenously) weekly or cisplatin $\left(80-100 \mathrm{mg} / \mathrm{m}^{2}\right)$ every 3 weeks during radiation.

\section{Follow-up}

The patients were evaluated for treatment response and adverse effect after IMRT as follows: every 2-3 months for the first 2 years, then every 3-4 months for years 3-5, and annually thereafter. The examination items included the following: physical examinations, flexible nasopharyngoscope, chest X-ray or CT, abdominal ultrasonography or CT, MRI of the head and neck, and a bone scan when necessary. The acute RT and chemotherapy related toxicities were assessed by the National Cancer Institute Common Toxicity Criteria (V.4.0). For evaluating the late adverse effects of RT, the Radiation Therapy Oncology Group and the European Organisation for Research and Treatment of Cancer were applied. ${ }^{8}$

\section{Statistical analysis}

Statistical analyses were performed with SPSS software (V.22.0). Specifically, OS was measured from the end of treatment to the observation of death caused for any reason; disease-free survival (DFS) was measured from the end of treatment to the first discovery of tumour recurrence or metastasis or death for any reason; local relapse-free survival (LRFS) and regional recurrence-free survival (RRFS) were measured from the end of treatment to the first observation of local recurrence and regional recurrence, respectively; distant metastasis-free survival (DMFS) was measured from the end of treatment to the observation of distant metastasis. The KaplanMeier method was used to draw survival curves and the $\log$ rank test was applied to compare differences. Multivariable analyses were conducted with a Cox proportional hazard model and the HR and the corresponding 95\% CI were calculated. Categorical and continuous variables were compared with a $\chi^{2}$ test and an independent t-test, respectively. In all cases, a two-sided $\mathrm{p}<0.05$ was considered to be statistically significant.

Because the patients and/or the public were not involved in the design, or conduct, or reporting, or dissemination plans of this study. Therefore, the Ethics Committee of Xijing Hospital approved the retrospective study, but did not provide an ethics number/ID.

\section{Patient and public involvement}

Patients and/or the public were not involved in the design, or conduct, or reporting, or dissemination plans of this study.

\section{RESULTS}

\section{The characteristics of patients}

A total of 792 patients were included. The distribution of the patients is presented in table 1. Overall, the median age was 47.3 years (with a range of 9-83 years) and the male $(\mathrm{n}=566)$-to-female $(\mathrm{n}=226)$ ratio was $2.5: 1$. The mean volume of primary tumour was $22.5 \mathrm{~mL}$ (with a range of 2.4-232 $\mathrm{mL}$ ). The mean diameter of metastatic cervical lymph nodes was $1.7 \mathrm{~cm}$ (with a range of $0.8-8.9$ $\mathrm{cm})$. The distribution of clinical stage was $22(2.8 \%), 124$ (15.7\%), $246(31.1 \%), 246(31.1 \%)$, and $154(19.4 \%)$ for stages I, II, III, IVa and IVb, respectively. The majority of patients were histologically diagnosed as WHO II $(\mathrm{n}=210 ; 26.5 \%)$ and WHO III $(\mathrm{n}=579 ; 73.1 \%)$, except for three patients who were diagnosed as WHO I $(n=3$; $0.4 \%)$. In this cohort, most patients were Han Chinese $(\mathrm{n}=772 ; 97.4 \%)$, followed by Hui People $(\mathrm{n}=15 ; 1.9 \%)$, Tibetan $(n=3 ; 0.4 \%)$, and Mongolian $(n=2 ; 0.3 \%)$. Only one of the ethnic minorities was histologically diagnosed as WHO type II, and the rest were all WHO type III. An MRI of the head and neck was selected as the diagnostic imaging technique for all patients. Nearly all patients $(93.9 \%)$ received chemotherapy, in various patterns, such as CCRT $(\mathrm{n}=243,30.7 \%)$, IC+CCRT $(\mathrm{n}=365 ; 46.1 \%)$, IC+RT $(\mathrm{n}=51 ; 6.4 \%)$, CCRT + AC $(\mathrm{n}=21 ; 2.7 \%), \mathrm{RT}+\mathrm{AC}$ $(\mathrm{n}=8 ; 1.0 \%), \mathrm{IC}+\mathrm{CCRT}+\mathrm{AC}(\mathrm{n}=55 ; 6.9 \%)$ and IC+RT+ AC $(n=1 ; 0.1 \%)$. The median follow-up time was 46.2 months (with a range of 1.3-130.2 months).

\section{Survival outcomes}

Overall, the 5-year LRFS, RRFS, DMFS, DFS and OS rates were $93.4 \%, 97.0 \%, 82.8 \%, 69.6 \%$ and $78.0 \%$, respectively (figure 2). There were significant differences in the DFS and $\mathrm{OS}$ rates between the subgroups of age, T-stage, $\mathrm{N}$-stage, clinical stage, histology, lactic dehydrogenase (LDH) and cervical nodal necrosis $(\mathrm{CNN})$. In addition, we found that tumour volume was associated with DFS and anaemia, with or without chemotherapy (CCRT, IC and AC) were related to OS. Significant differences in DMFS rates were observed between subgroups of N-stage, clinical stage, histology, tumour volume, CNN, Epstein-Barr virus (EBV)-DNA copy number, anaemia and with or without chemotherapy. Also, we found CNN and AC were associated with LRFS, and only IC was related to RRFS. Specifically, the patients with T4 disease had a marginally higher risk of local relapse than the patients with $\mathrm{T} 1$ disease $\left(\chi^{2}=1.699 ; \mathrm{p}=0.053\right)$. After clinical stage stratification by $\mathrm{N}$ stage, the RRFS rates were significantly lower in the N3 stage than in the N1 stage $\left(\chi^{2}=4.916\right.$; $\mathrm{p}=0.027$ ), while the differences were not significant between other subgroups (table 2).

\section{Multivariate analysis}

To do the multivariate analysis, statistically significant factors $(\mathrm{p}<0.1)$ of DFS, OS, DMFS, LRFS and RRFS rates in univariate analyses were enrolled into the Cox regression model. The results showed that age, N-stage, clinical stage, histology, the volume of primary tumour and LDH were independent prognostic factors for DFS. Concerning DMFS, we only 

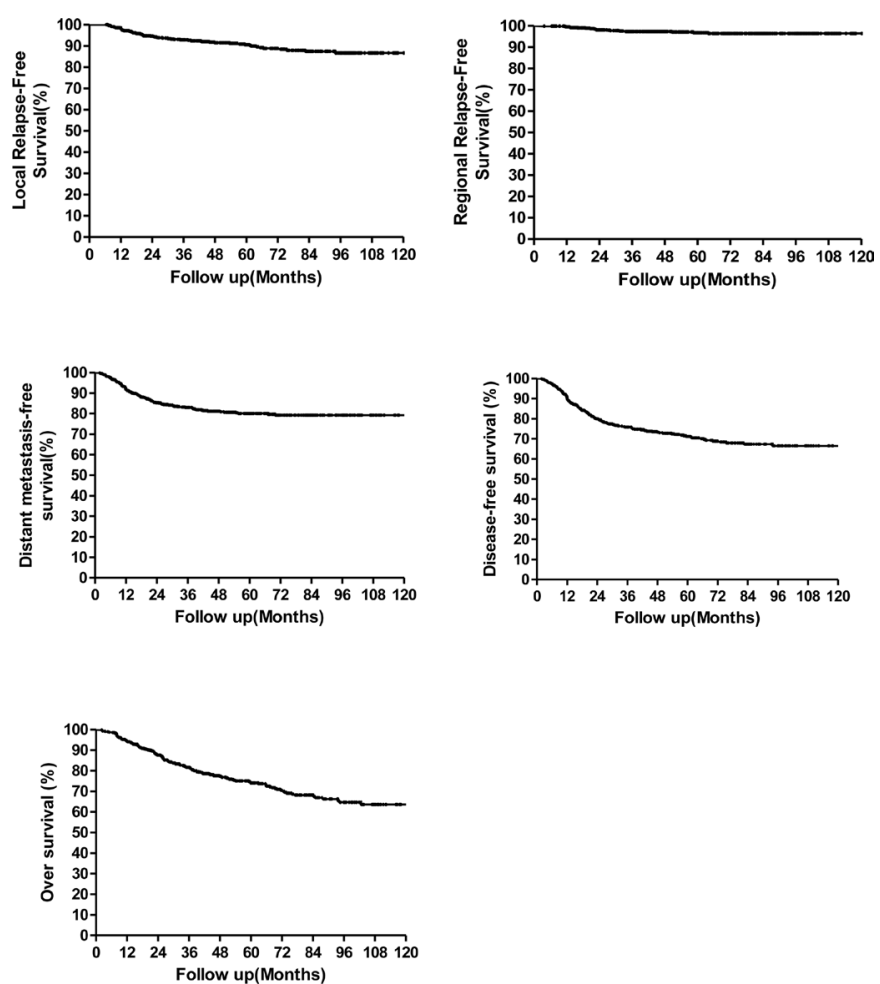

Figure 2 The local recurrence-free survival, regional recurrence-free survival, and distant metastasis-free survival, disease-free survival, and overall survival curves of patients who underwent IMRT. IMRT, intensity-modulated radiotherapy.

identified N-stage and cervical node necrosis (CNN) as the significant prognostic factors. Furthermore, we found that age, N-stage, histology, CNN and anaemia were significantly correlated with OS (table 3).

\section{Adverse effects}

There were 792 and 737 patients who were followed up for more than 1 year and were included to assess the acute and late chemo-RT-related toxicities, respectively (table 4). The most common acute toxicities for radiation were grade I and II dermatitis (534/792; 67.4\%), mucositis (520/792; 65.7\%) and dysphagia (632/792; 79.8\%). The most frequent late toxicity after treatment was xerostomia with occurrence rates of grade I 108 (14.6\%), grade II $354(48.15 \%)$ and grade III $108(14.6 \%)$. The incidence rate of xerostomia was significantly increased when combined with synchronous chemotherapy ( $79.3 \%$ vs $60.2 \%$; $\mathrm{p}=0.002)$. Grade I hearing impairment $(525 ; 71.2 \%)$ was the second most common late toxicity of IMRT. Likewise, combined cisplatin-based chemotherapy increased the incidence rate of hearing impairment caused by radiation $(80.6 \%$ vs $15.2 \%$; $\mathrm{p}<0.001)$. The main grade III acute toxicities of RT were dermatitis $(68 / 792$; $8.6 \%)$ and mucositis $(64 / 792 ; 8.1 \%)$. The only detected grade III acute toxicities of chemotherapy was neutropenia $(31 / 792 ; 3.9 \%)$. As for the late toxicities, only 108 patients (14.6\%) had grade III xerostomia. Remarkably, no severe grade intravenously toxicities were observed in our cohort.

\section{Failure patterns}

During the follow-up period, we observed 162 (20.5\%) deaths and $196(24.7 \%)$ treatment failures. A shown in table 5 , the major cause of failure was distant metastasis $(n=118 ; 60.2 \%)$, followed by local failure $(n=60 ; 30.6 \%)$, regional failure $(n=18 ; 9.2 \%)$. Concerning the causes of death, distant metastasis ranked the first, while other causes, such as RT or chemotherapy-related complications $(n=5)$, other malignant tumours $(n=1)$, no cancer causes $(n=3)$ and unknown causes $(n=2)$, only account for a tiny proportion of the deaths. In our cohort, $87.3 \%$ $(103 / 118)$ of patients developed distant metastasis within 3 years after treatment. The median time for the appearance of distant metastasis was 16.2 months (with a range of $0.8-68.3$ months). In patients with distant metastasis, $68(68 / 118,57.6 \%)$ had solitary metastasis to the bone, lung, liver, distant lymph nodes or parotid lymph nodes. Among these patterns, 4 (4/118; 3.4\%) had extra regional lymph node metastasis (axillary lymph node metastasis and mediastinal lymph node metastasis), and 2 (2/118; $1.7 \%$ ) had intraregional parotid lymph node metastasis. There were 45 patients $(45 / 118 ; 40.7 \%)$ developed two sites of metastasis, and the specific metastatic sites and cases are shown in table 6 . In addition, $78(9.8 \%)$ patients developed local or regional failures, with the median recurrence time of 27.0 months (range 4.4-92.3 months). The salvage treatments for these patients were reirradiation for 62 patients with local failures, surgery for three patients with regional failures, and palliative chemotherapy for patients who appropriate.

\section{The effect of chemotherapy}

We further evaluated the effect of combining chemotherapy with IMRT in NPC patients. The most frequently used strategies in our institution were IC plus CCRT $(\mathrm{n}=365 ; 46.1 \%)$ and CCRT $(\mathrm{n}=243 ; 30.7 \%)$. During IC, $72.4 \%(358 / 472)$ of patients were treated with docetaxelbased chemotherapy, while $15.0 \%(71 / 472)$ of patients received a gemcitabine-based regimen. The survival analyses demonstrated that there were no significant differences of LRFS, RRFS, DMFS and OS rates among these regimens of IC or AC. As for IC, specifically, the 5-year DFS and OS rates showed a trend of improving survival in the subgroup of TPF/TP as compared with other regimens, but significant differences were not achieved. In comparison of different $\mathrm{AC}$ regimens, these trends were not observed (table 7).

\section{DISCUSSION}

IMRT has been generally recognised as the standard radiation technique for NPC patients (NCCN guidelines for head and neck cancer, V.1, 2019). However, studies comparing the survival outcomes and adverse effects of NPC patients treated with IMRT between endemic and non-endemic regions are limited. In the current study, we reported an experience of IMRT for non-metastatic NPC in a non-endemic area of China (northwest China) based 


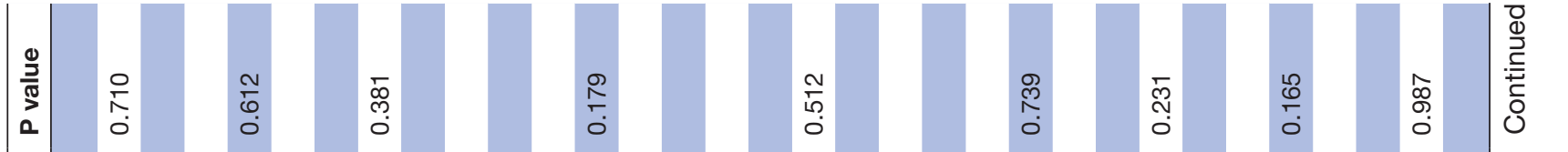

部部

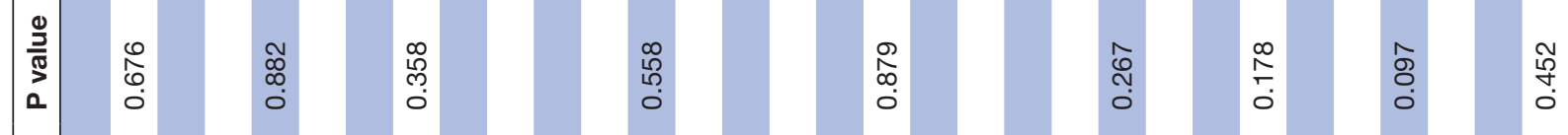

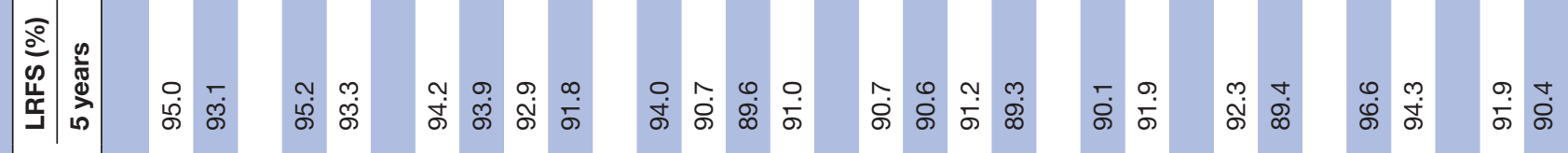

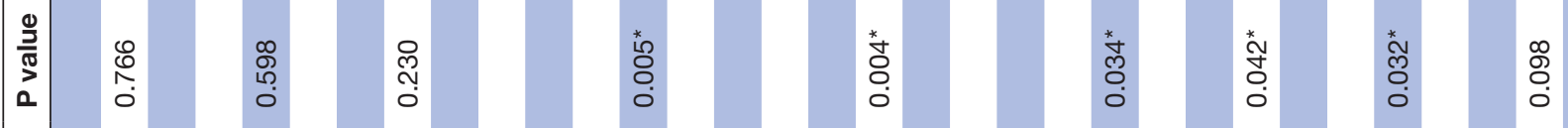

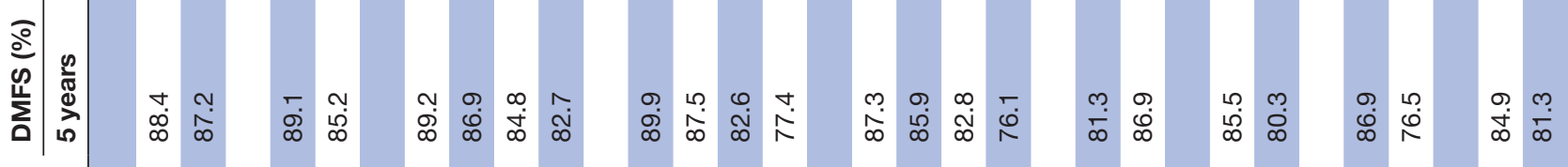

纹言

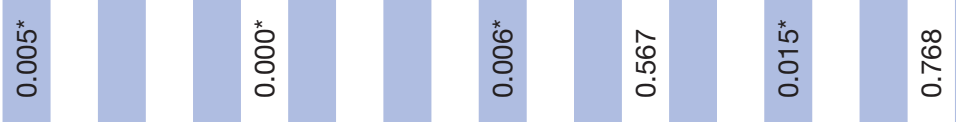

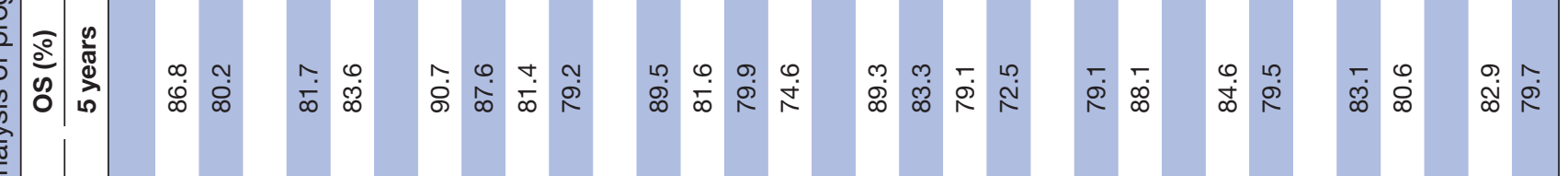

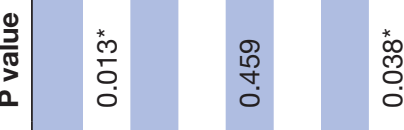

言

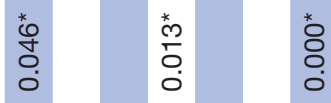

㕝

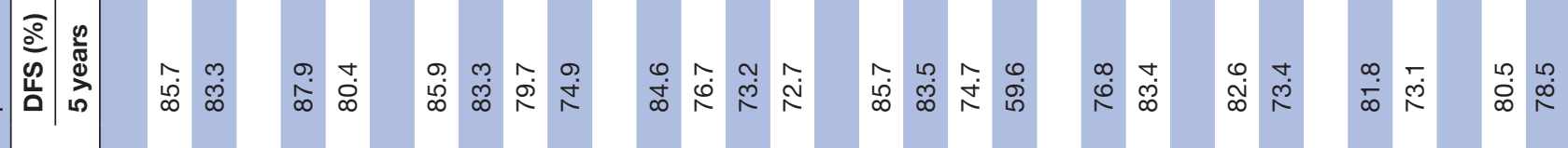

z

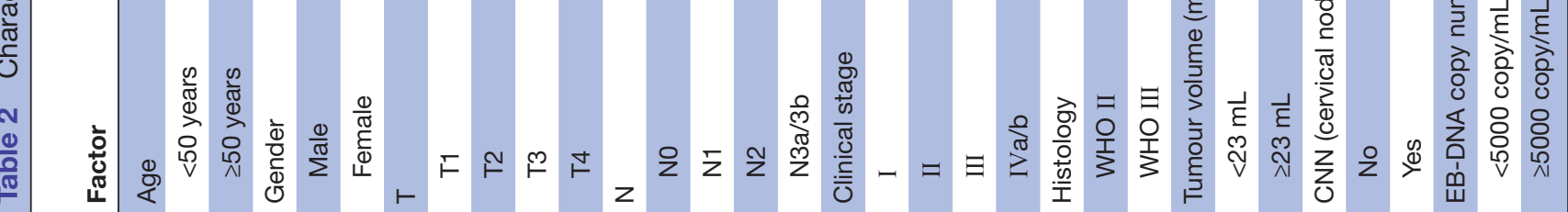




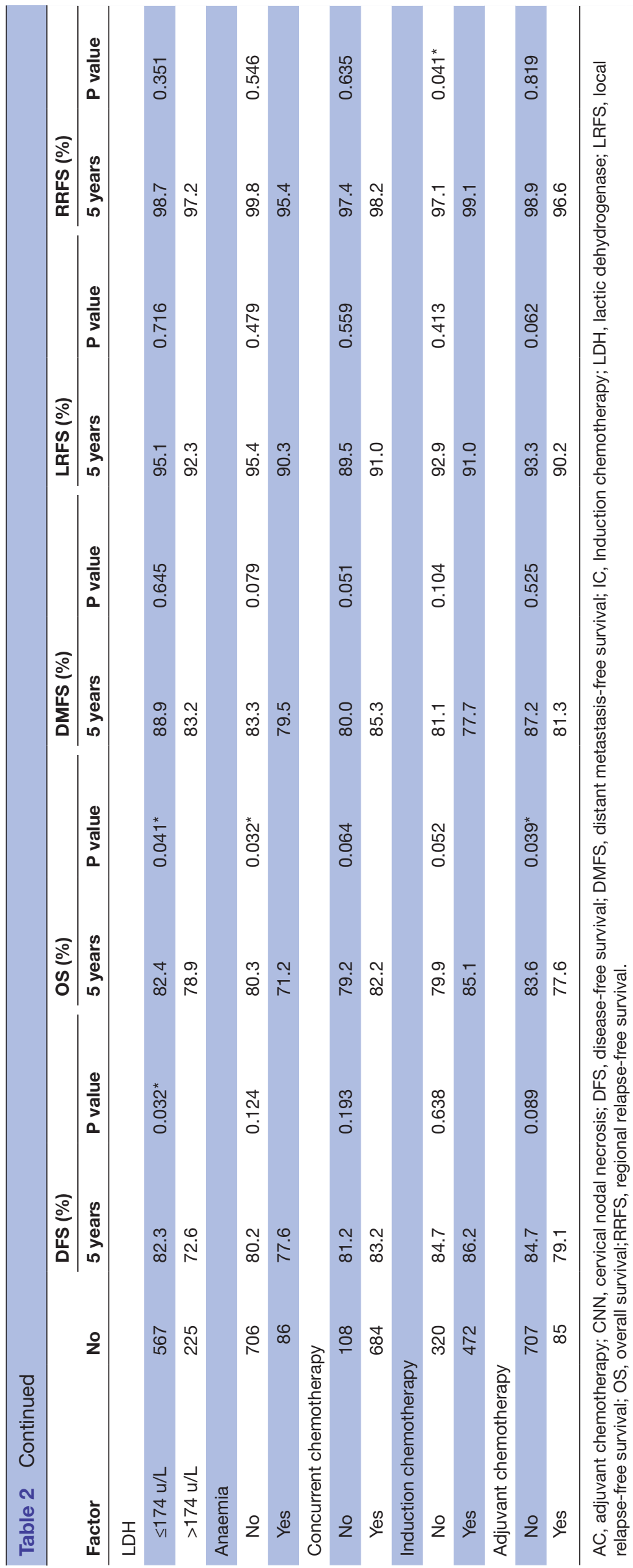

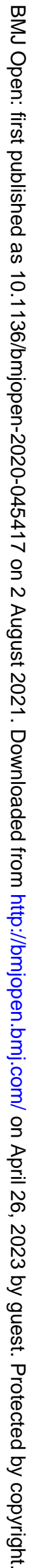


Table 3 Multivariate analysis of variables correlated with various clinical endpoints

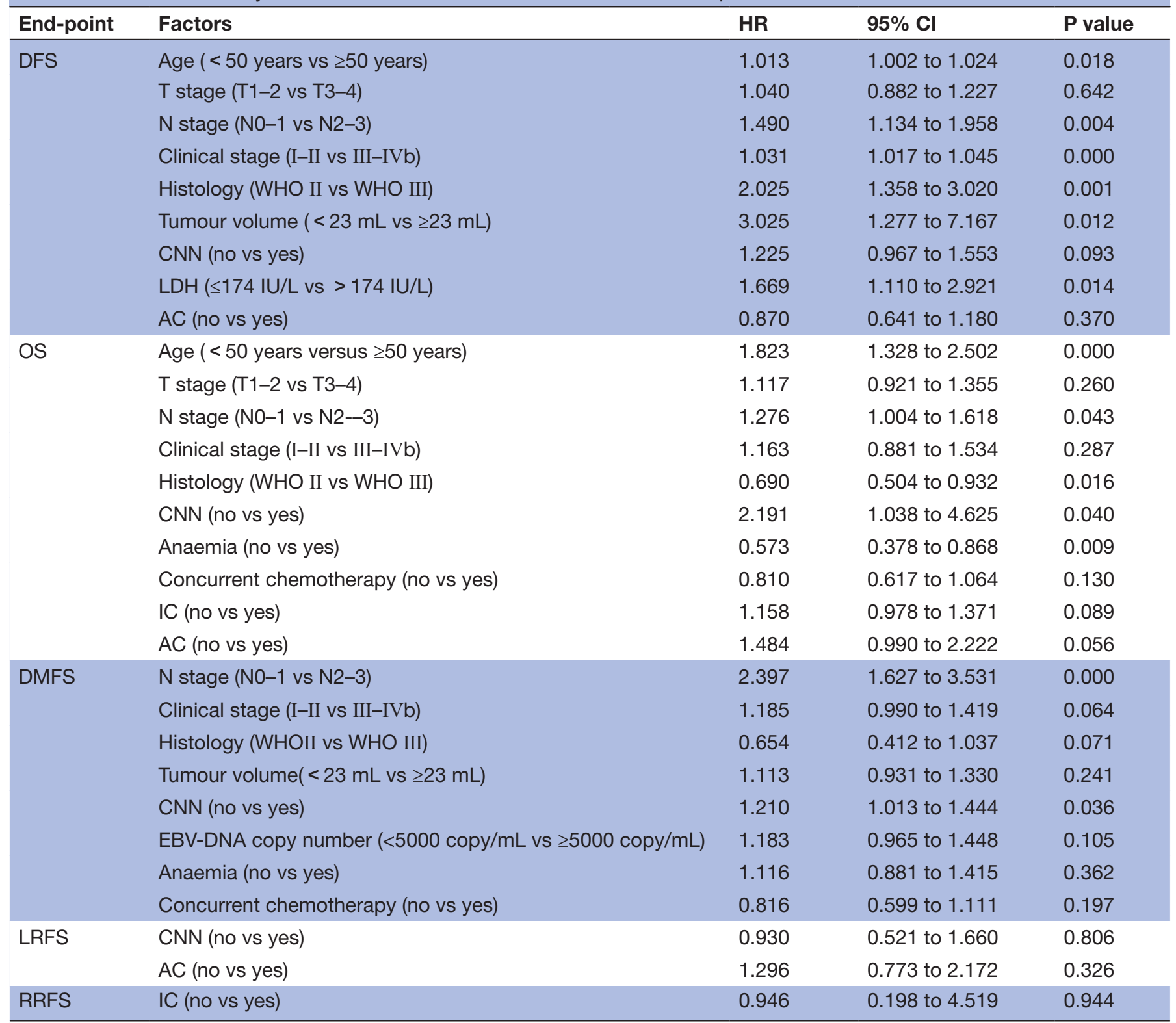

AC, adjuvant chemotherapy; CNN, cervical nodal necrosis; DFS, disease-free survival; DMFS, distant metastasis-free survival; IC, induction chemotherapy; LDH, lactic dehydrogenase; LRFS, local relapse-free survival; OS, overall survival; RRFS, regional relapse-free survival.

on a large cohort $(\mathrm{n}=792)$ and long follow-up time $(46.2$ months).

In recent years, literature has shown that IMRT was significantly associated with improved therapeutic effects of NPC patients. A prospective study enrolled 616 cases of non-metastatic NPCs (306 cases in the IMRT group and 310 cases in the 2D-CRT group) with a median follow-up time of 42 months to compare the survival outcomes. The results confirmed that IMRT was more effective than 2D-CRT. The 5-year LRFS and OS rates increased from $84.7 \%$ to $90.5 \%$ and $67.1 \%$ to $79.6 \%$, respectively. The IMRT-related toxicities were significantly lower than that of 2D-CRT. ${ }^{4}$ In a retrospective analysis, ${ }^{9} 527$ patients with NPC treated with IMRT achieved excellent survival outcomes; the 5-year LRFS, RRFS, DMFS, PFS and OS rates were $91.7 \%, 96.2 \%, 83.0 \%, 75.6 \%$ and $80.9 \%$, respectively. Tian et $a l^{10}$ reported the efficacy of IMRT in treating 865 NPC patients. After 10 years of follow-up, the LRFS, RRFS, DMFS, PFS, and OS rates were $92.0 \%$, $96.5 \%, 83.4 \%, 75.7 \%$ and $76.6 \%$, respectively.

However, the above results were all obtained from clinical centres in epidemic regions. Compared with the results of IMRT in epidemic regions, the survival outcomes obtained by our clinical centre in a non-endemic region of China were similar, except that the DFS and OS rates were slightly lower than that of endemic regions. The discrepancies may be due to several reasons. (1) the early diagnosis of NPC is difficult for its occult onset. Physicians in non-endemic regions particularly lack comprehensive knowledge and high vigilance for NPC. This results in 
Open access

Table 4 Treatment-related toxicities

\begin{tabular}{|c|c|c|c|c|c|}
\hline \multirow[b]{2}{*}{ Toxicities } & \multicolumn{5}{|c|}{ No of patients by toxicity grade (\%) } \\
\hline & 0 & 1 & 2 & 3 & 4 \\
\hline \multicolumn{6}{|c|}{ Acute toxicity related to radiotherapy } \\
\hline Dermatitis & $190(24.0)$ & $320(40.4)$ & $214(27.0)$ & $68(8.6)$ & $0(0)$ \\
\hline Mucositis & $208(26.3)$ & $300(37.9)$ & $220(27.8)$ & $64(8.1)$ & $0(0)$ \\
\hline Dysphagia & $160(20.2)$ & $516(65.2)$ & $116(14.6)$ & $0(0)$ & $0(0)$ \\
\hline \multicolumn{6}{|c|}{ Acute toxicity related to chemotherapy } \\
\hline Anaemia & $724(91.4)$ & $62(7.8)$ & $6(0.8)$ & $0(0)$ & $0(0)$ \\
\hline Thrombocytopenia & 699 (88.3) & $52(6.6)$ & $41(5.2)$ & $0(0)$ & $0(0)$ \\
\hline Neutropenia & $398(50.3)$ & $214(27.0)$ & $149(18.8)$ & $31(3.9)$ & $0(0)$ \\
\hline Febrile neutropenia & 747 (94.3) & $40(5.1)$ & $5(0.6)$ & $0(0)$ & $0(0)$ \\
\hline Vomiting & $238(30.0)$ & $476(60.1)$ & $78(9.9)$ & $0(0)$ & $0(0)$ \\
\hline Hand-foot syndrome & $0(0)$ & $0(0)$ & $0(0)$ & $0(0)$ & $0(0)$ \\
\hline Ototoxicity & $669(84.5)$ & $123(15.5)$ & $0(0)$ & $0(0)$ & $0(0)$ \\
\hline Neuropathy & $0(0)$ & $0(0)$ & $0(0)$ & $0(0)$ & $0(0)$ \\
\hline \multicolumn{6}{|c|}{ The late toxicities (737 patients) } \\
\hline Xerostomia & $167(22.7)$ & $108(14.6)$ & $354(48.1)$ & $108(14.6)$ & $0(0)$ \\
\hline Neck fibrosis & $716(97.2)$ & $21(2.8)$ & $0(0)$ & $0(0)$ & $0(0)$ \\
\hline Trismus & $723(98.1)$ & $14(1.9)$ & $0(0)$ & $0(0)$ & $0(0)$ \\
\hline Dysphagia & $680(92.3)$ & $39(5.3)$ & $18(2.4)$ & $0(0)$ & $0(0)$ \\
\hline Hearing impairment & $200(27.1)$ & $525(71.2)$ & $12(1.6)$ & $0(0)$ & $0(0)$ \\
\hline Temporal necrosis & $0(0)$ & $0(0)$ & $0(0)$ & $0(0)$ & $0(0)$ \\
\hline Cranial nerve palsy & 731 (99.2) & $6(0.8)$ & $0(0)$ & $0(0)$ & $0(0)$ \\
\hline
\end{tabular}

Table 5 Failure patterns of all patients

\begin{tabular}{lc}
\hline Variable & No. of patients (\%) \\
\hline Pattern of failure & \\
\hline Distant metastasis & $118(14.9)^{\star}$ \\
\hline Local and/or regional failures & $78(9.8)^{\star}$ \\
\hline Local failures alone & $60(7.6)$ \\
\hline Regional failures alone & $18(2.3)$ \\
\hline Local and regional failures & $9(1.1)$ \\
\hline Distant+local/regional failures & $15(1.9)$ \\
\hline Total & $196(24.7)$ \\
\hline Cause of death & \\
\hline Distant metastasis & $106(13.4)$ \\
\hline Local or regional failure & $45(5.7)$ \\
\hline $\begin{array}{l}\text { radiotherapy or chemotherapy- } \\
\text { related complications }\end{array}$ & $5(0.6)$ \\
\hline Other malignant tumours & $1(0.1)$ \\
\hline No cancer causes & $3(0.4)$ \\
\hline Unknown causes & $2(0.3)$ \\
\hline Total & $162(20.5)$ \\
\hline
\end{tabular}

*The number includes the 15 patents with both distant and local/ regional failures.
Table 6 Sites of distant metastasis $(n=118)$

\begin{tabular}{|c|c|}
\hline Site of distant metastasis & No \\
\hline \multicolumn{2}{|l|}{ Solitary } \\
\hline Bone & 50 \\
\hline Lung & 38 \\
\hline Liver & 36 \\
\hline Distant lymph nodes & 5 \\
\hline Parotid lymph nodes & 3 \\
\hline
\end{tabular}

Two sites

Bone and lung $\quad 15$

Bone and liver $\quad 12$

Lung and liver 8

Lung and distant lymph nodes 3

Liver and distant lymph nodes $\quad 1$

Parotid lymph nodes and distant lymph nodes 2

Epidural and spine 2

Multiple sites

Bone and lung and Liver 4

Others 
Table 7 The 5-year estimated survival rates stratified by various regimens of chemotherapy of locally advanced nasopharyngeal carcinoma

\begin{tabular}{lllll}
\hline 5y (No/\%) & $\begin{array}{l}\text { LRFS } \\
\text { (\%) }\end{array}$ & $\begin{array}{l}\text { RRFS } \\
\text { (\%) }\end{array}$ & $\begin{array}{l}\text { DMFS } \\
\text { (\%) }\end{array}$ & $\begin{array}{l}\text { OS } \\
(\%)\end{array}$ \\
\hline IC regimens (472) & & & & \\
\hline TPF/TP (67/291/27.4) & 95.7 & 97.8 & 82.3 & 90.6 \\
\hline GP (71/5.4) & 93.1 & 94.2 & 73.2 & 74.2 \\
\hline PF (29/2.2) & 90.6 & 93.0 & 76.3 & 71.3 \\
\hline Others (14/1.1) & 90.0 & 93.3 & 71.2 & 68.4 \\
\hline$\chi^{2}$ & 0.156 & 2.134 & 2.145 & 0.313 \\
\hline P value & 0.652 & 0.123 & 0.276 & 0.576 \\
\hline AC regimens (85) & & & & \\
\hline TPF/TP (11/22/38.8) & 90.6 & 92.1 & 71.8 & 77.7 \\
\hline GP (6/7.1) & 89.2 & 94.3 & 76.0 & 79.3 \\
\hline PF (30/35.3) & 88.1 & 100 & 71.4 & 74.1 \\
\hline Others (16/18.8) & 90.1 & 88.9 & 77.7 & 72.7 \\
\hline$\chi^{2}$ & 0.117 & 0.392 & 0.356 & 2.242 \\
\hline P value & 0.732 & 0.576 & 0.516 & 0.243 \\
\hline
\end{tabular}

AC, adjuvant chemotherapy; DMFS, distant metastasis-free survival; IC, induction chemotherapy; LRFS, local relapse-free survival; OS, overall survival; RRFS, regional relapse-free survival.

the higher percentage of $81.6 \%$ new cases diagnosed as stage III-IV in our centre compared with that reported in endemic regions of China, ${ }^{11}{ }^{12}$ (2) NPCs diagnosed in our centre usually have larger primary lesions and more severe cervical lymph node metastases. The average volume of nasopharyngeal tumours was $22.5 \mathrm{~mL}$, and the mean diameter of cervical lymph nodes was $1.7 \mathrm{~cm}$ in this cohort. A previous study in our centre has reported that the volume of the primary tumour of at least $23 \mathrm{~mL}$ was a poor prognostic factor for OS. ${ }^{13}$ Similarly, a study of 992 NPC patients treated with IMRT revealed that tumour volume was an independent prognostic factor for OS. ${ }^{14}$ In addition, the literature has shown that cervical lymph nodes necrosis was a significant prognostic factor for DMFS and $O S^{15}$ and (3) the number of NPC patients with WHO type II histology in our cohort is higher than that in epidemic regions. Studies have confirmed the close relationship of the WHO II pathological type with poor DFS, OS and DMFS. ${ }^{16-18}$

The tumour, node and metastasis (TNM) staging system, reflecting the extent of primary tumour invasion and regional lymph node involvement, plays a crucial role in the treatment of tumours and has the clinical value of guiding treatment response and predicting prognosis. With the advancement of radiation technology, the role of $\mathrm{T}$ stage on prognosis has been weakened, and only $\mathrm{N}$ stage remains a prognostic factor for non-metastatic NPC. ${ }^{19}$ Univariate analysis of our cohort demonstrated that $T$ stage was an independent prognostic factor for DFS and OS, while advanced $\mathrm{N}$ stage was an adverse prognostic factor for DFS, OS and DMFS. In the subgroup analyses, we showed that there was no significant difference in LRFS among T stages $\left(\chi^{2}=0.845\right.$; $p$-value $\left.=0.358\right)$, except that between T1 and T4 subgroups $\left(\chi^{2}=1.699\right.$; $\mathrm{p}=0.053)$. Similarly, the 5-year local control rates of $\mathrm{T} 1$ and T2 were both $94 \%$ in a previous study. ${ }^{20}$ The other study revealed that the LRFS rates were not significantly varied between patients with stages $\mathrm{T} 1$ and $\mathrm{T} 2$ and stages T2 and T3. ${ }^{21}$ Yang $e t a l^{22}$ reported that there were no statistical differences in RRFS between stages T2 and T3 and stages T2 and T4 ( $>0.05)$ when using the seventh edition Union for International Cancer Control (UICC)/American Joint Committee on Cancer (AJCC) staging system. However, a significant difference was observed in RRFS between stages T3 and T4 in the eighth edition staging system $(\mathrm{p}=0.001)$. These studies suggest that a more optimised TNM staging system is needed to better guide clinical practice and predict prognosis. The negative results of local control achieved by IMRT among various T stages are mainly due to the dosimetric advantages of the IMRT technique, which is sufficient even to treat stage $\mathrm{T} 4$ patients. After disease stratification by $\mathrm{N}$ stage, the 5-year DMFS of N0, N1, N2 and N3 were $89.9 \%, 85.7 \%, 82.6 \%$ and $77.4 \%$, respectively. With the increase in $\mathrm{N}$ stage, the DMFS rates declined progressively, and the difference was statistically significant $(p=0.005)$. Significant differences were not observed in RRFS among $\mathrm{N}$ stages, maybe due to the excellent regional control achieved by IMRT in all $\mathrm{N}$ stages (N0-3: 100\%, 97.1\%, 97.3\% and 93.5\%; $\mathrm{p}=0.179)$. This is similar to the results of previously reported literature. $^{1123}$

Apart from the TNM staging system, clinical parameters such as age, gender, histology and EBV-DNA copy number, LDHs are also potential prognostic factors for survival outcomes. In our data, age was an independent prognostic factor for DFS and OS. This result is controversial since age was not shown to be a poor prognostic factor in a previous study ${ }^{24}$ but has been reported as an independent prognostic factor in another study. ${ }^{25} \mathrm{In}$ addition, haemoglobin level of less than $110 \mathrm{~g} / \mathrm{L}$ before treatment was detected to be a poor prognostic factor for OS, which was consistent with previous results reported in our centre ${ }^{16}$ and a study published by another centre. ${ }^{26}$ Thus, dynamic monitoring of haemoglobin levels before and during RT and infusion of red blood cell suspension when necessary are of clinical benefits in improving the prognosis of NPC patients. The reason may be that treatment of anaemia has the potential to improve tumour hypoxia and further enhances radiation sensitivity. Additionally, the improvement of the nutritional status of patients can enhance their tolerance to chemo-RT. In our cohort, we detected a proportion of $50.6 \%$ of patients with definite cervical lymph node metastasis who simultaneously had lymph node liquefaction necrosis; the necrosis of lymph nodes was significantly associated with DMFS, DFS and OS. Consistently, Feng et $a l^{15}$ reported that necrosis of cervical lymph nodes was a poor prognostic factor for OS and DMFS. Our results indicate that more intensive treatments, such as those combined with IC, AC, and immune 
or targeted therapy, are needed for patients with stage N3 and with lymph node necrosis. High level of LDH ( $>174 \mathrm{IU} / \mathrm{L}$ ) was found to be associated with poor disease control in this study, which was consistent with the findings of previous studies. ${ }^{27}{ }^{28}$ However, multivariate analysis failed to select the LDH level as an independent prognostic factor in patients with WHO typeII, which warrants further prospective and large cohort studies to confirm the results in the future.

Regarding failure patterns, our results demonstrated that distant metastasis was the main mode of treatment failure. A majority of distant metastases occur within 3 years after treatment. The most common site of metastasis was bone, followed by lung and liver, which is similar to the data reported by other research centres. ${ }^{12}{ }^{29}$ In our cohort, $40.7 \%$ of patients had multiple organ metastases after treatment, which is consistent with the results in epidemic regions. ${ }^{30}$ While in a non-IMRT treatment modality, the most common observed failure mode was local recurrence. ${ }^{31}$ The reason could be that IMRT uses more precise immobilisation devices to make the error of treatment within a controllable range. Additionally, IMRT can obtain higher biological effects through the simultaneous-integrated boost technique. ${ }^{32}$ Due to the boosted and uniform doses of IMRT to the primary lesion and metastatic lymph node of NPC, the local and/or regional controls were strikingly enhanced. ${ }^{33}$ While the satisfactory local and/or regional controls have been achieved by IMRT, distant metastasis still needs to be further improved. Lai et $a P^{34}$ compared 512 NPC patients treated with IMRT and 764 patients treated with 2D-CRT; the DMFS was similar in both groups. This suggests that the role of IMRT in controlling the distant metastasis of NPC is limited. It is reported that ${ }^{35}$ the primary tumour cells may spread far away in the early or even precancerous stage of the tumour, forming an occult metastasis. When the body conditions are suitable, for example, in a state of immune deficiency or decline, the disseminated tumour cells will colonise in distant organs and form a premetastatic site. Therefore, how to use more advanced imaging techniques and laboratory examination methods to detect occult lesions may be one of the future directions for reducing the rate of distant metastasis of NPC.

Superior dosimetric advantage of IMRT facilitates the protection of organs at risk, thereby alleviating the adverse effects of patients. We demonstrated that the incidence rates of xerostomia, hearing impairment, cervical fibrosis and temporal lobe necrosis were similar to those reported in endemic regions. When combined with platinum-based chemotherapy, more severe hearing impairment and xerostomia were observed. Considerations should be made to select appropriate patients to receive appropriate chemotherapy regimens, for the sake of reducing the late oral and ear related toxicities and improving quality of life.

In the era of IMRT, the role of combined chemotherapy with IMRT has been constantly questioned and studied. The risk of death was declined to 0.79 and the 5-year OS rate was increased by $6.3 \%$ after CCRT followed by AC. ${ }^{36}$ Sun $^{11}$ analysed 868 locoregionally advanced NPC patients who received various treatment modalities and showed that there were no significant differences among survival outcomes. Our results showed that IC significantly increased RRFS (97.1\% vs $99.1 \%$; $\mathrm{p}=0.041)$ and OS $(79.9 \%$ vs $85.1 \%$; $\mathrm{p}=0.052)$, while AC had a survival benefit on OS (77.6\% vs $83.6 \%$; $p=0.039)$ and increased DFS (84.7\% vs $79.1 \%$; $\mathrm{p}=0.089)$ and LRFS with marginal significance $(93.3 \%$ vs $90.2 \% ; \mathrm{p}=0.062)$. In terms of chemotherapy regimens, docetaxel and gemcitabine based IC or AC showed a tendency to improve survival, which was consistent with the results of previous studies in our centre. ${ }^{18} \mathrm{~A}$ prospective study also reported that TPF-based regimens combined with CCRT significantly reduced the failure rate ( 3 year FFS: $80 \%$ vs $72 \%$; $=0.034$ ) and improved OS ( 3 year OS: $92 \%$ vs $86 \%$; $\mathrm{p}=0.029$ ) for locally advanced NPC. ${ }^{37}$

\section{Limitations}

Our study has several limitations. First, based on the characteristics of retrospective studies, we were unable to manually control the confounding variables, such as different induction or AC regimens. Hence, we conducted multivariate analyses to adjust for these confounding factors. Second, this was a single-centre study from a nonendemic region in China. A well-designed multicentre randomised controlled study is necessary to further explore the best treatment modality for newly diagnosed non-metastatic NPC in non-endemic region.

\section{CONCLUSIONS}

Based on a large cohort $(\mathrm{n}=792)$ and a long follow-up time (46.2 months), we revealed that the survival outcomes of NPC patients achieved by IMRT in the non-endemic region of China is comparable to that in endemic regions. The most common seen acute and late toxicities were similar to the patients treated in endemic regions. Distant metastasis and local/regional relapses were the top two patterns of failure.

Contributors MX, JZ and XL designed the study, conducted the statistical analysis and interpreted the results. MX, SL and JW collected the data. MX drafted the manuscript. All authors have read and approved the final version of the submitted manuscript.

Funding The authors have not declared a specific grant for this research from any funding agency in the public, commercial or not-for-profit sectors.

Competing interests None declared.

Patient and public involvement Patients and/or the public were not involved in the design, or conduct, or reporting, or dissemination plans of this research.

\section{Patient consent for publication Obtained.}

Ethics approval Approval of our study was granted by the Ethics Committee of Xijing Hospital, Air Force Military Medical University, Xian, China. Signed informed consent forms were kindly provided by each patient.

Provenance and peer review Not commissioned; externally peer reviewed.

Data availability statement Data are available on reasonable request. The datasets generated and analysed during the current study are not publicly available 
due to restricting patient privacy regulations by the different countries but are available from the corresponding author on reasonable request.

Open access This is an open access article distributed in accordance with the Creative Commons Attribution Non Commercial (CC BY-NC 4.0) license, which permits others to distribute, remix, adapt, build upon this work non-commercially, and license their derivative works on different terms, provided the original work is properly cited, appropriate credit is given, any changes made indicated, and the use is non-commercial. See: http://creativecommons.org/licenses/by-nc/4.0/.

ORCID iD

Xuqi Li http://orcid.org/0000-0002-2497-020X

\section{REFERENCES}

1 Ferlay J, Steliarova-Foucher E, Lortet-Tieulent J, et al. Cancer incidence and mortality patterns in Europe: estimates for 40 countries in 2012. Eur J Cancer 2013:49:1374-403.

2 Bray F, Ferlay J, Soerjomataram I, et al. Global cancer statistics 2018: GLOBOCAN estimates of incidence and mortality worldwide for 36 cancers in 185 countries. CA Cancer J Clin 2018;68:394-424.

3 Wei K-R, Zheng R-S, Zhang S-W, et al. Nasopharyngeal carcinoma incidence and mortality in China, 2013. Chin J Cancer 2017;36:90.

4 Peng G, Wang T, Yang K-Y, et al. A prospective, randomized study comparing outcomes and toxicities of intensity-modulated radiotherapy vs. conventional two-dimensional radiotherapy for the treatment of nasopharyngeal carcinoma. Radiother Oncol 2012;104:286-93.

5 Zhang B, Mo Z, Du W, et al. Intensity-Modulated radiation therapy versus $2 \mathrm{D}-\mathrm{RT}$ or $3 \mathrm{D}-\mathrm{CRT}$ for the treatment of nasopharyngeal carcinoma: a systematic review and meta-analysis. Oral Oncol 2015;51:1041-6.

6 Co J, Mejia MB, Dizon JM. Evidence on effectiveness of intensitymodulated radiotherapy versus 2-dimensional radiotherapy in the treatment of nasopharyngeal carcinoma: meta-analysis and a systematic review of the literature. Head Neck 2016;38 Suppl $1: E 2130-42$.

7 Mao Y-P, Tang L-L, Chen L, et al. Prognostic factors and failure patterns in non-metastatic nasopharyngeal carcinoma after intensitymodulated radiotherapy. Chin J Cancer 2016;35:103.

8 Cox JD, Stetz J, Pajak TF. Toxicity criteria of the radiation therapy Oncology Group (RTOG) and the European organization for research and treatment of cancer (EORTC). Int J Radiat Oncol Biol Phys 1995;31:1341-6.

9 Zhao W, Lei $\mathrm{H}$, Zhu X, et al. Investigation of long-term survival outcomes and failure patterns of patients with nasopharyngeal carcinoma receiving intensity-modulated radiotherapy: a retrospective analysis. Oncotarget 2016;7:86914-25.

10 Tian Y-M, Liu M-Z, Zeng L, et al. Long-Term outcome and pattern of failure for patients with nasopharyngeal carcinoma treated with intensity-modulated radiotherapy. Head Neck 2019;41:1246-52.

11 Sun X, Su S, Chen C, et al. Long-Term outcomes of intensitymodulated radiotherapy for 868 patients with nasopharyngeal carcinoma: an analysis of survival and treatment toxicities. Radiother Oncol 2014;110:398-403.

12 Ou X, Zhou X, Shi Q, et al. Treatment outcomes and late toxicities of 869 patients with nasopharyngeal carcinoma treated with definitive intensity modulated radiation therapy: new insight into the value of total dose of cisplatin and radiation boost. Oncotarget 2015;6:38381-97.

13 Zang J, Li C, Zhao L-N, et al. Prognostic model of death and distant metastasis for nasopharyngeal carcinoma patients receiving 3DCRT/ IMRT in nonendemic area of China. Medicine 2016;95:e3794.

14 Chen C, Fei Z, Huang C, et al. Prognostic value of tumor burden in nasopharyngeal carcinoma. Cancer Manag Res 2018;10:3169-75.

15 Feng Y, Cao C, Hu Q, et al. Prognostic value and staging classification of lymph nodal necrosis in nasopharyngeal carcinoma after intensity-modulated radiotherapy. Cancer Res Treat 2019;51:1222-30.

16 Wang J, Shi M, Hsia Y, et al. Failure patterns and survival in patients with nasopharyngeal carcinoma treated with intensity modulated radiation in northwest China: a pilot study. Radiat Oncol 2012;7:2.

17 Zhao L-N, Zhou B, Shi M, et al. Clinical outcome for nasopharyngeal carcinoma with predominantly who II histology treated with intensity- modulated radiation therapy in non-endemic region of China. Oral Oncol 2012;48:864-9.

18 Zhao L, Xu M, Jiang W, et al. Induction chemotherapy for the treatment of non-endemic locally advanced nasopharyngeal carcinoma. Oncotarget 2017;8:6763-74.

19 Lin S, Lu JJ, Han L, et al. Sequential chemotherapy and intensitymodulated radiation therapy in the management of locoregionally advanced nasopharyngeal carcinoma: experience of 370 consecutive cases. BMC Cancer 2010;10:39.

20 Lee AWM, Ng WT, Chan LK, et al. The strength/weakness of the AJCC/UICC staging system (7th edition) for nasopharyngeal cancer and suggestions for future improvement. Oral Oncol 2012;48:1007-13.

21 Zong J, Lin S, Lin J, et al. Impact of intensity-modulated radiotherapy on nasopharyngeal carcinoma: validation of the 7th edition AJCC staging system. Oral Oncol 2015;51:254-9.

22 Yang X-L, Wang Y, Liang S-B, et al. Comparison of the seventh and eighth editions of the UICC/AJCC staging system for nasopharyngeal carcinoma: analysis of 1317 patients treated with intensitymodulated radiotherapy at two centers. BMC Cancer 2018;18:606.

23 Kam MKM, Teo PML, Chau RMC, et al. Treatment of nasopharyngea carcinoma with intensity-modulated radiotherapy: the Hong Kong experience. Int J Radiat Oncol Biol Phys 2004;60:1440-50.

24 Wang $\mathrm{R}$, Wu F, Lu H, et al. Definitive intensity-modulated radiation therapy for nasopharyngeal carcinoma: long-term outcome of a multicenter prospective study. J Cancer Res Clin Oncol 2013;139:139-45.

$25 \mathrm{Xu} \mathrm{L,} \mathrm{Pan} \mathrm{J,} \mathrm{Wu} \mathrm{J,} \mathrm{et} \mathrm{al.} \mathrm{Factors} \mathrm{associated} \mathrm{with} \mathrm{overall} \mathrm{survival}$ in 1706 patients with nasopharyngeal carcinoma: significance of intensive neoadjuvant chemotherapy and radiation break. Radiother Oncol 2010;96:94-9.

26 Zhang L-L, Zhou G-Q, Li Y-Y, et al. Combined prognostic value of pretreatment anemia and cervical node necrosis in patients with nasopharyngeal carcinoma receiving intensity-modulated radiotherapy: a large-scale retrospective study. Cancer Med 2017;6:2822-31.

27 Nie M, Sun P, Chen C, et al. Albumin-to-Alkaline phosphatase ratio: a novel prognostic index of overall survival in cisplatin-based chemotherapy-treated patients with metastatic nasopharyngeal carcinoma. J Cancer 2017;8:809-15.

28 Huang T, Su N, Zhang X, et al. Systemic chemotherapy and sequential locoregional radiotherapy in initially metastatic nasopharyngeal carcinoma: retrospective analysis with 821 cases. Head Neck 2020;42:1970-80.

29 Huang C-L, Guo R, Li J-Y, et al. Nasopharyngeal carcinoma treated with intensity-modulated radiotherapy: clinical outcomes and patterns of failure among subsets of 8th AJCC stage IVA. Eur Radiol 2020;30:816-22.

30 Sun X-S, Liu S-L, Luo M-J, et al. The association between the development of radiation therapy, image technology, and chemotherapy, and the survival of patients with nasopharyngeal carcinoma: a cohort study from 1990 to 2012. Int J Radiat Oncol Biol Phys 2019;105:581-90.

31 Cheng $\mathrm{SH}$, Yen KL, Jian JJ, et al. Examining prognostic factors and patterns of failure in nasopharyngeal carcinoma following concomitant radiotherapy and chemotherapy: impact on future clinical trials. Int J Radiat Oncol Biol Phys 2001;50:717-26.

32 Withers HR. Biological aspects of conformal therapy. Acta Oncol 2000;39:569-77.

33 Hara W, Loo BW, Goffinet DR, et al. Excellent local control with stereotactic radiotherapy boost after external beam radiotherapy in patients with nasopharyngeal carcinoma. Int J Radiat Oncol Biol Phys 2008;71:393-400.

34 Lai S-Z, Li W-F, Chen L, et al. How does intensity-modulated radiotherapy versus conventional two-dimensional radiotherapy influence the treatment results in nasopharyngeal carcinoma patients? Int J Radiat Oncol Biol Phys 2011;80:661-8.

35 Yeh AC, Ramaswamy S. Mechanisms of cancer cell dormancy-another hallmark of cancer? Cancer Res 2015;75:5014-22.

36 Blanchard P, Lee A, Marguet S, et al. Chemotherapy and radiotherapy in nasopharyngeal carcinoma: an update of the MACNPC meta-analysis. Lancet Oncol 2015;16:645-55.

37 Sun Y, Li W-F, Chen N-Y, et al. Induction chemotherapy plus concurrent chemoradiotherapy versus concurrent chemoradiotherapy alone in locoregionally advanced nasopharyngeal carcinoma: a phase 3, multicentre, randomised controlled trial. Lancet Oncol 2016;17:1509-20. 